\title{
A Survey on Hexapod Walking Robot and Gait Planning*
}

\author{
Qingyun Liu ${ }^{1, a^{*}}$, Tiantian Jing ${ }^{2, b}$ \\ ${ }^{1}$ School of Mechanical Engineering, Anhui University of Technology, Ma'anshan 243002, China \\ ${ }^{2}$ School of Mechanical Engineering, Anhui University of Technology, Ma'anshan 243002, China \\ aemail: lqyahjx@ahut.edu.cn, bemail: tiantianjing2015@163.com
}

Keywords: Hexapod walking robot ; Gait planning ; Obstacle avoidance ; Path planning.

Abstract. A survey on hexapod walking robots and gait planning is presented. For the discrete landing points, the redundant DOF of legs and the impending body, the performance of hexapod walking robots is better than wheeled robots and tracked robots. The prototypes of hexapod walking robots and its up-to-date development are introduced. The gait planning of the hexapod walking robot is discussed and the selection of landing points in the unstructured environment is summarized. The developing tendency and some further research contents in this domain are indicated.

\section{Introduction}

In the nature, there are some places that people can not arrive, e.g, volcanoes, mineral exploration, planetary exploration, rescue mission, terrain recognition, automation process, and dangerous waste cleanup and so on. In order to explore and research those environments, a series of approaches and ways have been searching all the time. The coming of robots, such as the wheeled robot, the tracked robot and the multi-legged walking robot, will be helpful for resolving those troubles. The research indicates that the wheeled robot can moves quickly on the relatively flat terrain for its simple structure and control system. However, the violent vibration, walking lability and energy consumption may appears and its sport efficiency will decreased seriously on the muddy, rough and soft terrain. The caterpillar of tracked robots can cover more areas and adapt to the soft, rough terrain, but it removes relatively slow, flexible bad and shaky seriously [1]. The multi-legged walking robot includes quadruped robots, hexapod robots and octopod robots. The hexapod robot is common. Compared with the wheeled robot and the tracked robot, the hexapod walking robot is a kind of mobile mechanism with the redundant actuation, the multi branched chain and the time varying topology. It imitates hexapod insects. Therefore, spacial properties of the hexapod robot are listed as follows: (1) its motion trajectory is a series of discrete landing points. It can choose the best support point to fall when walking on the ground and has good environmental adaptability and high mobility; (2) The legs have more degree of freedoms. The body's balance is kept by adjusting its position and the length of legs. The position of the center of gravity is also adjusted so that it can not easy to tilt; (3) The body is floated so that the robot can avoid the vibration caused by the terrain; (4) It could move quite quickly on the uneven pavement and the soft terrain and its energy consumption is low [2].

Based the characteristics of the hexapod walking robot, it has a wide of implementation in the military transportation, submarine detection, mine exploring, plant detection, education, entertainment and so on. The multi-legged walking robot technology has been a hot spot of researching at the domestic and foreign in the field of robotics. In this paper, the research results in the prototype design and the gait planning of the hexapod walking robot, and aspects to be further studied are systematically analyzed and summarized.

\section{Research Status of Hexapod Walking Robots}

The research inspiration of most hexapod walking robots comes from the natural insect.

The Biobot was introduced by Delcomyn [3] in 2000, who referred to the American cockroach, shown in Fig. 1. The whole size of this robot is $58 \mathrm{~cm} \times 14 \mathrm{~cm} \times 23 \mathrm{~cm}$ and its body and the leg size are 
12 to 17 times as large as the true cockroach. The structure and the ratio are similar to the true cockroach: the length ratio of hip, meropodium and tibia is 1:1:1.5. The standing height to the ground is $15 \mathrm{~cm}$. In order to make this robot move flexibly on the rough ground like insects, the pneumatic artificial muscle is chosen as the actuator. The compressed air is transmitted by a pipe on the upper part of the machine and drives the joints rotate, which imitates the performance of muscles. So, the pneumatic artificial muscle actuator can support greater force and higher speed than other robots, which actuated by the electricity and the hydraulic pressure.

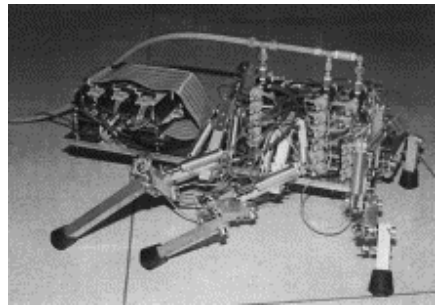

Fig. 1 Biobot.

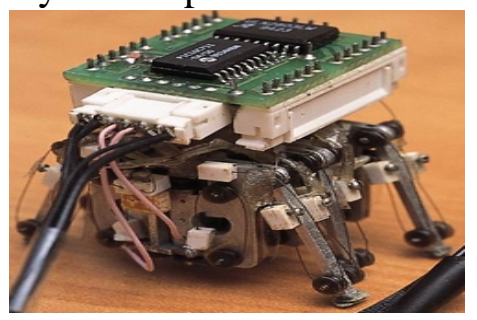

Fig. 2 SMA Robot-

Jie Zhu [4] proposed the SMA (Shape Memory Alloy) robot, which has two degrees of freedom in each leg. Shown in Fig. 2. This robot only can move along the straight line and cannot make a turn. In order to realize the turning, researchers improved the main body of this robot. The main body is made up two parallel trigeminal stents which connected by a bush. The SMA robot can finish the omnidirectional motion through the combined bias SMA actuator. The hexapod robot SHeRo [5], which its center of gravity can remove up and down, studied by Mahdi Agheli. It can be used to check, restore and fix the bridge, ship, pipe and storage tank because its body can across the ditch flexibly, shown in Fig. 3. Michael Henrey [6] developed the hexapod crawling robot Abigaille-III based on the gecko, shown in Fig. 4. This robot has the good suck force, grasping force and magnetic force as the double layer adhesive in the foot sole. For the important thing is that it can walk 4 hours continuously in the smooth or the rough vertical ground. The actuator system is consist of 24 micro gear motors and there are three or four motors in each leg. In a word, Abigaille-III fills the vacancy that the hexapod walking robot can not move in the vertical plane.

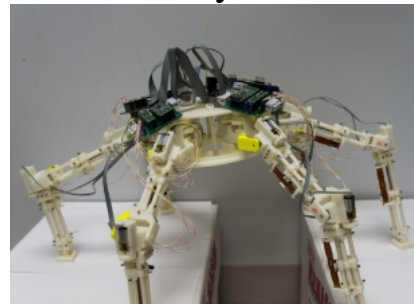

Fig. 3 SHeRo Robot.

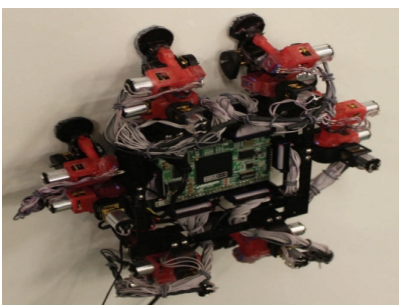

Fig. 4 Abigaille-III Robot.

Axel Schneider [7] introduced a new hexapod robot, HECTOR (HExapod Cognitive auTonomously Operating Robot), which combines a rich sensorization, compliant joint drives and decentral control approaches. Dong Liu [8] presented a hexapod robot with a neck joint installed between the front and central part in the body.

In an attempt to explore the irregular surface of plant and other dangerous places, a hexapod crawling intelligent robot, Genghis [9], which based the vision technology, is developed by MIT, shown in Fig. 5. This robot has more than 150 sensors that can be used to know kinds of information about outside and itself. The function of the only camera is to cross obstacles in the unknown environment.

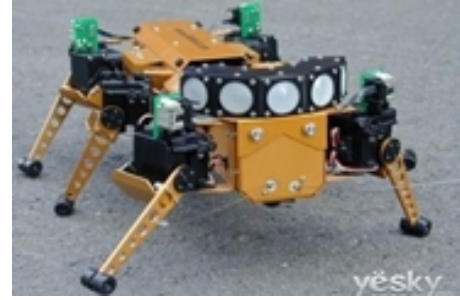

Fig. 5 Genghis Robot.

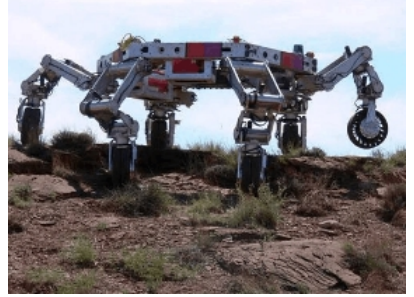

Fig. 6 ATHLETE Robot.

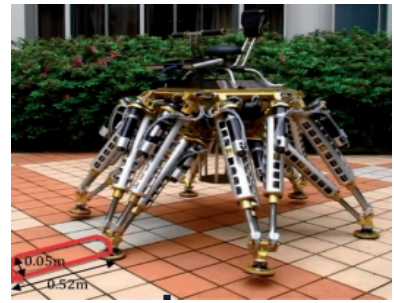

Fig. 7 Octopus Robot. 
In order to realize the strong carrying capacity of the hexapod walking robot, the ATHLETE [10], developed by the National Aeronautics and Space Administration (NASA), has six legs with wheels, shown in Fig. 6. Those wheels can fix the robot on the ground and help it climb out the mud to analyze the surface features or jump cross the big rock. Besides, this robot can climb the sleep slope with 36 degree obliquity and could down successfully. Meanwhile, the ATHLETE can load things with 15 tons. When on the horizontal surface, it can move fast and its legs can adapt varieties of complex terrains. Yang Pan [11] developed the Octopus robot, shown in Fig. 7. The body of this robot is the hexagon and the structure of the leg is parallel. The loading ability is $200 \mathrm{~kg}$. The leg is controlled by the combination of remote person and machine and can work in the extremely complex risk environment.

Except the land hexapod walking robot, there are numerous underwater robots play an important roles in exploring ocean.

In order to solve the issue of the shallow water mine, the Autonomous Legged Underwater Vehicle[12], which imitates the crab, is produced by the Luokeweier company in American, shown in Fig. 8. The each leg of this robot has two degrees of freedom and has the performance of amphibious movement. It can hide under the ocean waves and the foot can embedding in the sand in case washed away.

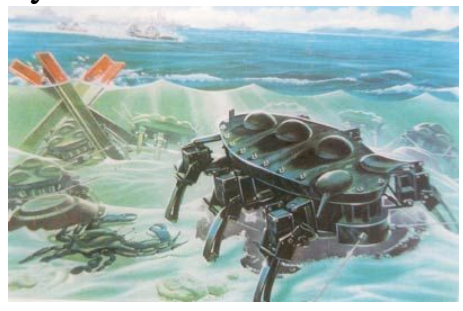

Fig. 8 ALUV Robot.

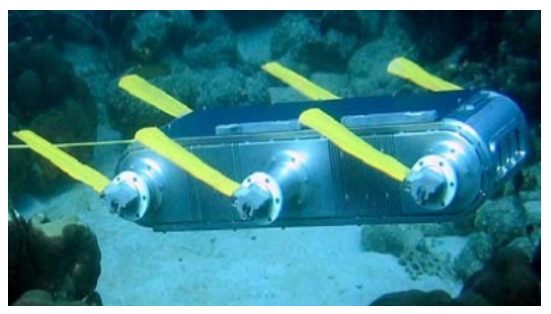

Fig. 9 the AQUA Platform.

Prahacs C [13] described the evolution of RHex that can move both sea and land and created three visions of RHex with increasing levels of aquatic capability. Three visions include the Research-RHex, the Shelley-RHex and the Rugged-RHex. The AQUA was developed based on the Rugged-RHex, which can swim to a depth of 10m, shown in Fig. 9. Haldum Komsuoglu [14]studied a relationship between energetic efficiency and the dynamical structure of the legged robot's gait by collecting the date set from a RHex-class machine. They found that tripod gaits of the RHex robot are dynamic and this dynamic behavior can get higher locomotion efficiency not only via increasing speed but also via decreasing power consumption.

The Whegs $\square$ in [15][16], a whole terrain adaptive humanoid robot, is researched by the Case Western Reserve University and the United State Naval Institute, shown in Fig. 10. Its leg is the three wheel leg mechanism and adopts the flexible mechanism design. There is a 60 degrees phase difference between the adjacent legs. This robot can walk in the water and ground with the tripod gait. When across the obstacle, the gait can be changed from the different gait to the same gait, which can enhance the ability of obstacle crossing. Besides, the spiral wheel could promote it move in the water and accomplish the rising and the diving through the body joint positive and negative rotation.

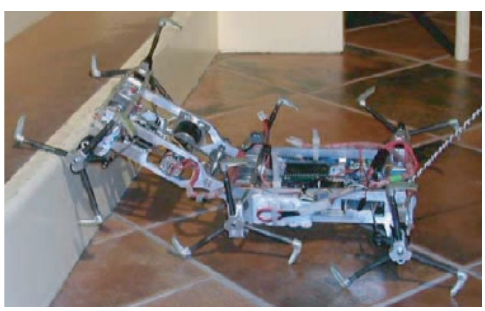

Fig. 10 Whegs Robot.

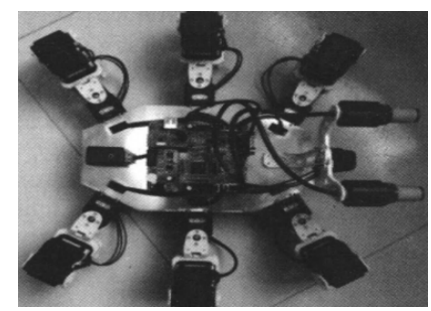

Fig. 11 Bionic Beetle Hexapod Robot.

In the material of robots, Shuhai Jiang [17] designed a bionic beetle hexapod robot, which has three degrees of freedom in each leg. The body is the octagon and six legs are on the both sides. All leg joints are driven by the servo motor and the joint is soft as the synthetic plastics replaces the metal. The material of robot body is the high-strength aluminum alloy material and the connection of the leg and the joint is the high-strength plastics. This way can decrease the weight and is good 
for running, shown in Fig. 11. Inspired robots's skin can provide a proper friction in forward motion and protect robots from slipping on the slope surface during locomotion, Poramate Manoonpong [18] designed a hexapod robot AMOS applied real shark skin to its each foot soles. The study shows that the AMOS can grip specific surfaces and move effectively without slipping.

In the control system of hexapod robots, in [19], the first implementation of the neurobiologically-based leg control method in hexapod robots is presented and this type robot can walk and navigate on uneven terrain by using this controller. Shown in Fig. 12, the BILL-Ant-a (Biologically-Inspired Legged Locomotion-Ant-autonomous) with the neurobiologically-based leg control walks autonomously and adaptively on irregular terrain. In [20], a hexapod robot with the servomotor control interface is studied, shown in Fig. 13. Roberto Sepúlveda and Oscar Montiel in [21] mentioned a fuzzy logic algorithm to control the hexapod robot in the " $x-y$ " plane body by using the genetic algorithm toolbox from Matlab.

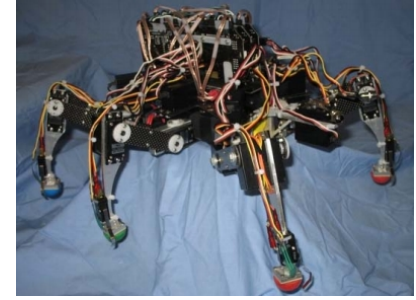

Fig. 12 the BILL-Ant-a Robot.

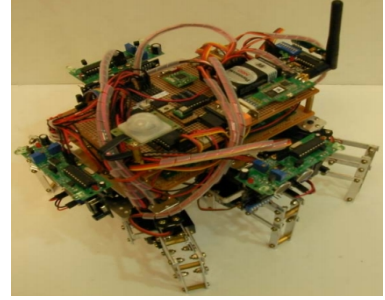

Fig. 13 the Completed Hexapod Robot.

\section{Gait Planning of the Hexapod Walking Robot}

The gait planning of each hexapod walking robot is diverse for the different structures. The gait is the walking way and rule to coordinate the operation of each leg. Because there are more degree of freedoms of each leg, the dynamics and the kinematics are very complex. The gait planning and the foot coordination control are the key points to research.

Triangle Gait: The triangle gait is that two groups leg (each group is composed of the propodium, the metapedes in one side and the mesopodium in another side ) are in the swing phase or the stance phase respectively. Three legs of each group makes up a triangle and their action are uniformed. So, there is only one group leg can walk at the same time: the propodium pulls the body; the mesopodium holds up the body; the metapedes pushes and turns the body. The action of walking is completed through the move of three legs and the alternation of two groups leg [22].

Wettergreen [23] made a freedom gait research based on the free action control to the Dante $\square$ robot; Jun Su [24] planned the straight line gait and the fixed turning gait theoretically through analyzing the kinematic characteristics of the robotic mechanical structure. At the same time the maximum stride and angle are confirmed. However, they only did the simulation results but the practical test is absent. Wei Feng and Xiangjun Qi [25][26] did a hexapod robot can finish the practical straight line gait and fixed turning gait flexibly by using the fischertechnik bionic robot package and can avoid many small obstacles. But, the gait disorder is inevitable because of existing the mismachining tolerance and the differences of motor performance. In view of the above matters, the photoelectric switch is used as the gait detection sensor of the hexapod walking robot by Jianhai Han [27]. The signal from the photoelectric switch can timely adjust the operative condition of the motor and correct the gait imbalance caused by the mechanical manufacturing error. Finally, this robot can not only obey the specified gait to walk, but also improve the walking efficiency and the service life.

Slope Gait: The robot may encounter the rough or the slope terrain in realistic situations. In an attempt to research and overcome the influence from the complex ground, Shuanzhu Li [28] designed the high stability margin of the slope gait from the joint angle of robot and enhanced the walking steady. But he ignored the effect of the speed, the load capacity, the external force and the center of gravity when the robot climbs the slope. Tianhong Liu [29] proposed the minimum stable distance. This method considered the influence of the external force, the external force, the height 
of the center of gravity, and the quality on the walking stability of the robot. It lays the foundation for the research of the soft robot.

Obstacle Avoidance Gait: Up to now, the walking gait of the most hexapod robots is the vertical triangle gait. In order to avoid obstacles efficiently, Lianrui Liu [30] put forward a transverse triangle gait, shown in Fig. 14. Even though this gait can avoid obstacles efficiently, the flexibility and sensitivity is not perfect and not suitable for emergency situations. Wu Wei [31] researched the hexapod climbing robot based on the transverse triangle gait, which is used for the bridge detection, tunnel detection and other special situations. Besides, they proposed the transverse four angular gait and it has the good maneuverability and security, shown in Fig. 15. From the program designing, Phuc [32] studied an algorithm about the robot climbing over small obstacles. But they did not provide the detailed algorithm process and how to over the big obstacle, too. Hema [33] provided the detailed algorithm and made robot across the big obstacle steadily. However, the method of identifying the obstacle is not introduced.

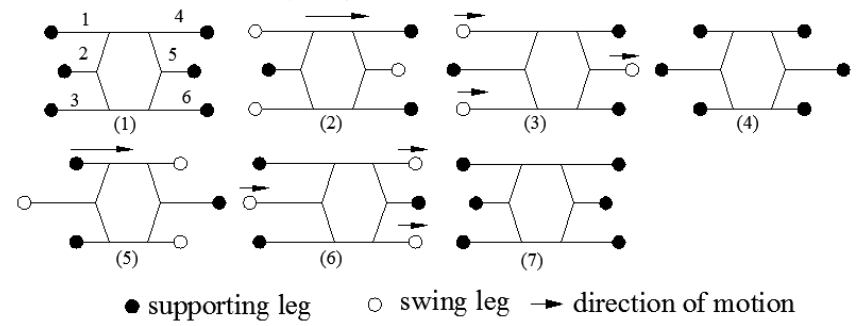

Fig. 14 The transverse triangle gait.

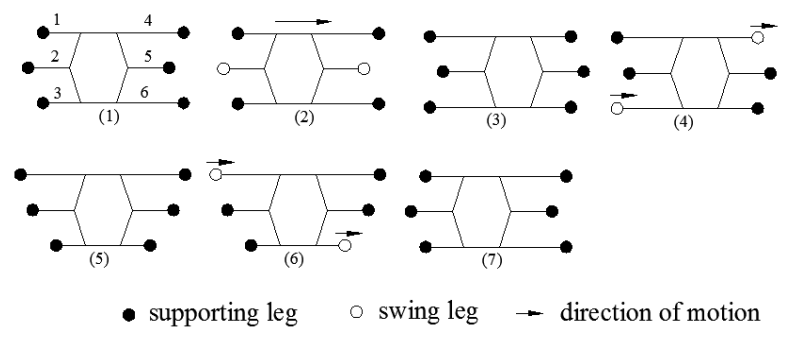

Fig. 15 The transverse four angle gait.

Fault-tolerant Gait: The leg of multi-legged robots could suffer a failure and be fail to operate the assigned task when they walk. Therefore, the fault-tolerant gait have an important role in gait planning. As a novel project of gait study, the fault-tolerant gait planning has drawn the attention of scientists and the notion of leg failure has been embedded into the frame of gait planning. In the prior works [34][35], the researcher has presented the fault-tolerant gait planning as hexapod robots against the occurrence of joint failure and proposed the corresponding principles. But those investigations has restrictions which hexapod robots only can walk on the perfect terrain. In [36], a novel fault-tolerant gait planning applied in the rough terrain, the Follow-The-Leader (FTL)gaits, is proposed. It makes hexapod robots traverse the rough ground at the same time maintain both fault-tolerance and gait stability. Besides, the motion of hexapod robots over the inclined plane and a general fault tolerant algorithm were discussed in [37].

\section{Path Planning and Obstacle Recognition}

Foot Path Planning: In the process of the multi-legged robot walking, the landing point selection and the trajectory planning have the important effect on the consistency, the stability, the beauty and so on. For the hexapod bionic walking robot, the ideal foot point trajectory should has better characteristics in taking-off and landing, speed and acceleration.

The common foot point trajectory includes the straight line, the parabola, the cardioid and the spline curve and so on. They can be described through the elementary function and the polynomial interopolation. Using the the elementary function to describe the trajectory will result the acceleration mutational phenomenon, which can influence the walking steady and lead to the overload of motors. The linear function can form a given shape, but it usually cause the uncontinuity of the line connection point between the acceleration and the velocity. The Starting angle and termination angle of the parabola could be kept and they are good for avoiding the obstacle. But the impact phenomenon exists when robot legs up and down [38]. There are the invariability of the step size and the step height as well as the little angle in the cardioid and the spline curve. The polynomial interopolation can satisfy the first order and two order guiding and continuation. But the degree of the polynomial interopolation can be overhigh and the shake and unsteady will be generated when samples are chosen too many [39][40]. At last, researcher find that the cubic spline curve can not only meet the curve first order and two order guiding and continuation, but also the trajectory is smooth and the guidance is decreased [41]. According to how 
adjust the downing point condition, Longhai Zhao [42] provided a adjustment strategy based on the downing point condition of robots. This strategy can be used for the motion planning of multi-legged walking robots at a full range in the natural terrain and adapt to kinds of gaits.

Obstacle Recognition: Mohe [43] provided a vision algorithm can recognize obstacles to some extent, but they ignored the transmission and the processing speed of the signal. So the practicality is poor. Choi [44] proposed a laser recognition algorithm based on the laser principle. This algorithm can identify the obstacle precisely and is not vulnerable to the environment. However, the distance of laser irradiation is related to the electric power, the power supply could be required. Budiharto [45] researched a voice controlled recognition algorithm can be used indoor, where noise is weak. But this method will be interfered severely in outside. Jenog [46] presented the thermal imaging recognition algorithm but it is easily affected by the environment. Bo You Error! Reference source not found. provided a gait planning strategy of the obstacle self recognition from the kinematics. This strategy can feedback to robots according to the landing time and the force touching ground time of legs. It can help the robot judge whether across the obstacle or not. So, this method has a good application.

\section{Conclusions}

Since the late twentieth century, the domestic and foreign scholars have developed a lot of robot experiment models and principle prototypes. But in general, the basic technology of the hexapod walking robot is difficult to support the large-scale practical application. For the hexapod walking robot, the contents is to be further researched as follows:

(1)Comprehensiveness of the gait planning. At present, Most of the researches on the gait of walking robots are focused on the conventional terrain. There are few researches about the matter of soil, swamp and relatively loose terrain. The matter of mud off and automatic recovery from the tumbling down in the muddy and the swamp are studied little.

(2)Similarity of the bionic structure. The most of asymmetrical legs of natural insects are different. The triangular structure of asymmetrical legs has lots of advantages, e.g. stable the center of gravity, easily over obstacles and flexible turning around. However, the majority of walking robots has the same size legs. So, it is impossible for the asymmetrical legs walking robot to become the trend of researching.

(3)Flexibility of the structure design. At present, most of the walking robot's legs are rigid, which will not only inevitably produce the collision and interference, but also make the poor walking stability, the serious friction and the poor life. In order to avoid those troubles, the design of the soft leg will lay the foundation for walking steadily.

(4)Simplification of the control algorithm. The complex body structure and numerous joints can result the sophisticated control method. So, the control algorithm of the simple robot will be a hot researching pot.

(5)Intelligence of the gait planning. The existing gait planning of multi-legged robots is studied based on kinematics (including forward kinematics and inverse kinematics) and the known surroundings. Hence, how to make robots realize the gait generating and location accurately after recognizing the unknown environment become very important.

\section{Acknowledgements}

This paper is supported by the Key Project of Provincial Natural Science Research, Dept. of Education for Anhui Province (KJ2014A028).

\section{References}

[1] Zhenbang Gong. The Mechanical Design of Robot. Beijing: Electronics Industry Press, 1995, 11. ( In Chinese ) Reference to a book. 
[2] Jing Liu, Xiaoguang Zhao, Min Tan.: submitted to Journal of Robot, 2006, 28(1), pp. 81-88.( In Chinese )

[3] Delcomyn F, Nelson M E.: submitted to Journal of Robotics and Autonomous Systems, 2000, 30, pp. 5-15.

[4] Jie Zhu, Zhikui Cao, Peisun Ma, etal.: submitted to Journal of Drive System Technique, 2002, 4, pp. 11-15. ( In Chinese )

[5] Mahdi Aghelin, Long Qu, Stephen S, Nestinger.: submitted to Journal of Robotics and Computer-Integrated Manufacturing, 2014, 30, pp. 478-488.

[6] Michael Henrey, Ausama Ahmed, Paolo Boscariol, etal.: submitted to Journal of Bionic Engineering, 2014, 11, pp. 1-17.

[7] Axel Schneider, Jan Paskarbeit, Malte Schilling, and Josef Schmitz.: submitted to Journal of Living Machines 2014, Springer International Publishing Switzerland, 2014, pp. 427-429 .

[8] Dong Liu, Weihai Chen, Zhongcai Pei. etal:: submitted to Journal of Mechanism and Machine Science 33. Springer International Publishing Switzerland, 2015, pp. 133-144.

[9] Rodney A. Brooks.: submitted to Journal of Neural Computation, 1989, 1(2), pp. 253-262.

[10] Information on http://www.4908.cn/html/2012-02/21335.html.

[11] Yang Pan, Feng Gao.: submitted to Proceedings of the Institution of Mechanical Engineers, Part C: Journal of Mechanical Engineering Science, 2014, 228(12), pp. 2217-2232. ( In Chinese )

[12] Greiner H, Shectman A.: submitted to Journal of Autonomous Underwater Vehicle Technology, 1996(6), pp. 41-48.

[13]Prahacs C, Saunders A, Smith M K, etal.: submitted to Proceedings of the Inaugural Canadian Design Engineering Network (CDEN) Design Conference[C]. Montreal Canadian: 2004.

[14]Haldun Komsuoglu, Anirudha Majumdar and Yasemin Ozkan Aydin, etal.: submitted to Journal of Springer Tracts in Advanced Robotics 79 (2014), pp. 667-684.

[15]Boxerbaum A S, Werk P, Quinn R D, etal.: submitted to Proceedings of the 2005 IEEE/ASME International Conference on Advance Intelligent Mechatronics [C]. Piscataway, NJ, USA: IEEE, 2005, pp. 1459-1465.

[16] Harkins R, Ward J, Vaidyanathan R, etal:: submitted to Proceedings of the 2005 IEEE/ASME International Conference on Advance Intelligent Mechatronics [C]. Piscataway, NJ, USA: IEEE, 2005, pp. 1465-1470.

[17] Shuhai Jiang, Pei Sun, Jingjing Tnag, etal:: submitted to Journal of Nanjing Forestry University (Natural Science Edition), 2012, 36(6), pp. 116-120. ( In Chinese )

[18]Poramate Manoonpong, Dennis Goldachmidt, Florentin Worgotter, etal.: submitted to Journal of Living Machines 2013. Springer-Verlag Berlin Heidelberg, 2013, pp. 402-404.

[19]Lewinger, W.A.: submitted to Proceedings of IEEE/RSJ International Conference on Intelligent Robots and Systems(2010), 1, pp. 3386-3391.

[20]Elsevier Ltd.: submitted to Journal of Robotic and Computer-Integrated Manufacturing (2012), 28(12), pp. 351-358.

[21]Roberto Sepúlveda, Oscar Montiel, Rodolfo Reyes, etal.: submitted to Journal of Computational Intelligence. Springer International Publishing Switzerland 2014, pp. 709-721.

[22] Xuedong Chen, Yu Sun, Wenchuan Jia. Motion Planning and Control of Multilegged Walking Robots[M]. Huazhong University of Science and Technology press, 2006. ( In Chinese ) Reference to a book

[23] Wettergreen D, Thorpe C.: submitted to Proceeding of 1996 the IEEE International Conference on Robotic and Automation. 1996, pp. 2718- 2723. 
[24]Jun Su, Xuedong Chen,Wengang Tian.: submitted to Journal of Mechanical and electronic, 2004, 3, pp. 48-52. ( In Chinese )

[25] Wei Feng, YangYang.: submitted to Journal of Machine Design and Research, 2005, 21(3), pp. 35-37. ( In Chinese )

[26]Xiangjun Qi, Lin Chen, Mingdan Liu.: submitted to Journal of Computer Simulation. 2007, 24(4), pp. 158-161. ( In Chinese )

[27]Jianhai Han, Shushang Zhao, Jishun Li.: submitted to Journal of Mechanical Electrical Engineering Magazine, 2004, 21(4), pp. 8-10. ( In Chinese )

[28] Shuanzhu Li, Jianfeng Zhong, Pin Cheng.: submitted to Journal of Mechanical and Electronic, 2013, 3, pp. 53-57. ( In Chinese )

[29] Tianhong Liu, Shuhai Jiang.: submitted to Journal of Computer Simulation, 2013, 30(12), pp. 360-364. ( In Chinese )

[30]Lianrui Liu, Ze Zhang, Jianhua Gao.: submitted to Journal of Zhejiang Sci-Tech University, 2011, 28(2), pp. 225-228. ( In Chinese )

[31] Wu Wei, Weili Dai.: submitted to Journal of Computer Engineering and Design, 2013, 34(1), pp. 268-272. ( In Chinese )

[32]Nguyen Dang Phuc, Nguyen Truong Thinh.: submitted to Proceedings of the 2011IEEE International Conference on Robotics and Biomimetcs. 2011, pp. 1707-1711.

[33] Hema, Paulraj and Abdul, etal.: submitted to Proceedings of 2011 IEEE Student Conference on Research and Development. 2011, pp. 117-122.

[34] Yang, J.M.: submitted to Journal of Robotica, 2005. 23(6), pp. 701-708.

[35] Yang, J.M.: submitted to Journal of Robotica, 2006. 24(2), pp. 269-270.

[36]JUNG-Min Yang.: submitted to Journal of Intell Robot Syst (2009), 54, pp. 613-627.

[37] Debabrata Sarkar, Shakti Kumar Dubey, Abhijit Mahapatra, etal.: submitted to Proceedings of 2th International Conference on Innovations in Automation and Mechatronics Engineering, ICIAME 2014, 14, pp. 93-99.

[38] Manhong Li, Jianhua Zhang, Minglu Zhang.: submitted to Journal of China Mechanical Engineering. 2014, 25(6), pp. 821-825. ( In Chinese )

[39] Monan Wang, Liquan Wang, Qingxin Meng, etal.: submitted to Journal of Harbin Engineering University, 2003, 24(2), pp. 180-183. ( In Chinese )

[40]Fu Chen, Xizhe Zang, Jie Zhao, etal.: submitted to Journal of Mechanical and Electronic, 2009, 9, pp. 53-56. ( In Chinese )

[41] Shangbin Run, Baoling Han, Qingsheng Luo.: submitted to Journal of Computer Simulation, 2007, 24(10), pp. 156-160. ( In Chinese )

[42]Longhai Zhao, Yubin Liu, Jie Zhao, etal.: submitted to Journal of Jiangnan Universitey (Natural Science Edition), 2013, 12(4), pp. 385-389. ( In Chinese )

[43]Zalhan Mohd Zin, Ratnqwati Ibrahim.: submitted to Proceedings of SPIE-The International Society for Optical Engineering. 2011.

[44]Baehoon Choi, Heejin Lee, KwangWoong Yang. etal.: submitted to Proceedings of 2012 International Conference on Fuzzy Theory and Its Applications, 2012, pp. 220-222.

[45]BUDIHARTO.: submitted to ITB Journal of Engineering Service, 2012, 44(2), pp. 148-167.

[46]Jeong Hyo Ha, Sijing Kim, Myung Jin Chung.: submitted to Proceedings of 2012 12th International Conference on Control, Automation and System. 2012, pp. 2268-2270. 
International Forum on Energy, Environment Science and Materials (IFEESM 2015)

[47]Bo You, Yufei Liu, Haibo Gao, etal.: submitted to Journal of Harbin University of Science and Technology, 2013, 18(5), pp. 42-46. ( In Chinese ) 